\title{
Contribuciones científicas presentadas en los congresos de la Asociación Española de Investigación de la Comunicación (2007-2018)
}

\section{Papers presented at the conferences of the Spanish Association for Communication Research (2007-2018)}

\author{
Gloria Gómez-Escalonilla; Patricia Izquierdo-Iranzo
}

\section{Cómo citar este artículo:}

Gómez-Escalonilla, Gloria; Izquierdo-Iranzo, Patricia (2020). "Contribuciones científicas presentadas en los congresos de la Asociación Española de Investigación de la Comunicación (2007-2018)". Profesional de la información, v. 29, n. 4, e290440.

https://doi.org/10.3145/epi.2020.jul.40

Artículo recibido el 09-03-2020

Aceptación definitiva: 28-04-2020

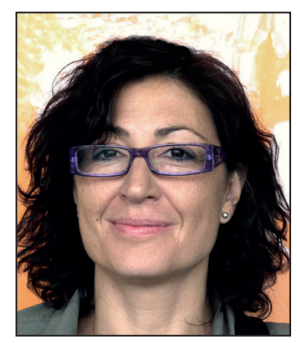
Gloria Gómez-Escalonilla $\bowtie$ https://orcid.org/0000-0003-1708-9665
Universidad Rey Juan Carlos Facultad de Comunicación Depto. de Ciencias de la Com. y Sociología Camino del Molino, 5. 28943 Fuenlabrada (Madrid), España gloria.gomezescalonilla@urjc.es

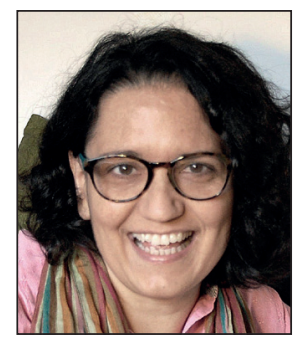
Patricia Izquierdo-Iranzo https://orcid.org/0000-0001-9429-2822

Universidad Rey Juan Carlos Facultad de Comunicación Depto. de Ciencias de la Com. y Sociología Camino del Molino, 5. 28943 Fuenlabrada (Madrid), España patricia.iranzo@urjc.es

\section{Resumen}

Se analiza el contenido de los 847 trabajos presentados por autores de universidades españolas en las 6 conferencias de la Asociación Española de Investigación en Comunicación (AE-IC). Las 6 conferencias se llevaron a cabo en Santiago de Compostela 2008, Málaga 2010, Tarragona 2012, Bilbao 2014, Madrid 2016 y Salamanca 2018. Las variables de investigación de estudio son: perfiles del autor, ubicación geográfica, género, universidad de origen, temas estudiados más comunes y medios involucrados. Los resultados confirman que ya no existe división de género: de hecho, hay más autoras que autores. La Universidad Complutense de Madrid y la Universitat Autònoma de Barcelona son las que tienen las tasas de participación más altas, y Madrid y Catalunya son las regiones más activas, seguidas por Andalucía y Valencia. Los temas tratados son bastante diversos, destacando los estudios de contenidos de los medios, principalmente los centrados en la comunicación política. Otros temas comunes son el impacto de la era digital y la estructura de los medios audiovisuales.

\section{Palabras clave}

Investigación en Comunicación; Meta-investigación; Análisis de contenido; Congresos científicos; Asociación Española de Investigación en Comunicación; AE-IC; Ponencias; Comunicaciones; Producción científica; Universidades; Temas de investigación; Brecha de género.

\footnotetext{
Abstract

The content of the 847 papers presented by authors from Spanish universities at the six conferences of the Spanish Communication Research Association (AE-IC) is analyzed. The six conferences were held in Santiago de Compostela (2008), Malaga (2010), Tarragona (2012), Bilbao (2014), Madrid (2016), and Salamanca (2018). The research variables of study are: author's profiles, geographic location, gender, home university, most commonly studied topics, and considered media. The results confirm that the gender divide no longer exist: in fact, there are more female than male authors. Universidad Complutense de Madrid and Universitat Autònoma de Barcelona exhibit the highest rates of participation, and Madrid and Catalonia are the most active regions, followed by Andalusia and Valencia. The topics treated are quite diverse, with the study of media content standing out, mainly those focused on political communication. Other common topics are the impact of the digital age, and audiovisual media structure.
} 


\section{Keywords}

Communication research; Meta-research; Content analysis; Academic conferences; Spanish Communication Research Association; Conference papers; Scientific production; Universities; Research topics; Gender divide.

\section{Introducción}

Conocer cómo es la investigación sobre una disciplina es una aproximación válida para ampliar el conocimiento científico sobre la misma, lo cual contribuye a su construcción social al aportar datos, enfoques e interpretaciones que ayudan a entenderla de otra manera. El estudio de las prácticas investigadoras constituye un campo de estudio: la metodología de la ciencia, con líneas de investigación propias en cada disciplina. Los condicionantes históricos, sociales y culturales también las definen, como bien ha señalado la sociología del conocimiento.

En las ciencias de la Comunicación existe una línea de investigación asentada y con cierta tradición que toma como objeto de estudio precisamente la propia investigación en Comunicación. La meta-investigación en España como línea propia cuenta con algunas referencias pioneras a finales del siglo pasado (De-Moragas, 1988; Caffarel-Serra; Domínguez; Romano, 1989; Cáceres; Caffarel-Serra, 1993; Urabayen, 1994; Jones, 1998), pero no es hasta bien entrado el presente siglo cuando se afianza en consonancia con el nivel de madurez del campo de estudio. Hoy día existe una amplia cartera de trabajos muy completos que han ido cartografiando el sistema de investigación nacional en Comunicación: (Almirón; Reig, 2007; Piñuel-Raigada; Lozano-Ascencio; García-Jiménez, 2011; Casado; Fernández-Quijada, 2013; Díaz-Nosty; De-Frutos-García, 2016; Saperas-Lapiedra, 2016), incluso por áreas específicas (Baladrón-Pazos; Correyero-Ruiz; Manchado-Pérez, 2014) como la publicidad o desde perspectivas particulares, como el estudio crítico que coordinaron Rodríguez-Serrano y Gil-Soldevilla en 2018.

En esta línea de investigación se sitúa el proyecto de investigación «El sistema de investigación en España sobre prácticas sociales de Comunicación: Mapa de proyectos, grupos, líneas, objetos de estudio y métodos» (CSO2013-48933-C4) desarrollado en el período 2007-2013 y el proyecto "Mapa de la Investigación en Comunicación en las Universidades Españolas de 2007 a 2018" (PGC2018-093358-B-100) que actualmente se está llevando a cabo, actualizando los datos del mapa de la investigación de la Comunicación: http://www.mapcom.com

El presente artículo forma parte de este proyecto competitivo abordando el análisis de las contribuciones aportadas a los congresos organizados por la Asociación Española de Investigadores de la Comunicación desde su fundación en 2007 hasta 2018, seis congresos científicos que, aunque no contienen toda la investigación en Comunicación que se hace en España, sí consideramos que pueden ser representativos del trabajo de los investigadores y docentes españoles.

La meta-investigación sobre Comunicación en nuestro país ha investigado la actividad realizada por los grupos de investigación (Tur-Viñes; Núñez-Gómez, 2018), las tesis doctorales (Jones; Baró, 2000; Marcos-Recio; Martínez-Pestaña; Blasco-López, 2012; Castillo-Esparcia; Xifra, 2006) o los proyectos I+D (Pacios; Vianello-Osti; Rodríguez-Bravo, 2016; Barranquero-Carretero; Limón-Serrano, 2017). También hay bibliografía sobre las editoriales especializadas (Giménez-Toledo; Tejada-Artigas, 2012), el efecto de las agencias evaluadoras en la producción de los autores (Jiménez-Contreras; De-Moya-Anegón; Delgado-López-Cózar, 2003) que también es tratado críticamente en la esfera internacional (Aalbers, 2004; Barker, 2007; Pusser; Marginson, 2013; Banfi; Franzini; Galimberti, 2014) o incluso el papel de las redes sociales y la publicación/visibilidad científica (Orduña-Malea; Martín-Martín; Delgado-López-Cózar, 2016; Arcila-Calderón; Calderín-Cruz; Sánchez-Holgado, 2019), cuestión recurrente así mismo en la bibliografía internacional (Harzing; Alakangas, 2016). Mención especial merece el estudio de las revistas, acorde al lugar protagónico que ocupan como vehículo difusor de la labor investigadora (Gross; Harmon; Reidy, 2002; Braun; Dióspatonyi, 2005). Esta proliferación permite una alta especialización y se analiza desde la internacionalidad de los autores españoles (Fernández-Quijada; Masip, 2013; Costa-Sánchez, 2017), o las estrategias de las coautorías (Fernández-Quijada; Masip, 2013; Escribá-Sales; Cortiñas, 2013), hasta los efectos psicológicos que produce la presión por publicar (Goyanes; Rodríguez-Gómez, 2018), pero la mayoría de trabajos sobre los artículos se centran en aspectos bibliométricos (Castillo-Esparcia; Carretón-BaIlester, 2010; Gorraiz, 2018; Repiso; Orduña-Malea; Aguaded, 2019). Frente a esta variedad y abundancia, contrasta la escasez de referencias dedicadas a analizar en detalle las ponencias de los congresos, encontrando a modo testimonial el trabajo de Rodríguez-Gómez (2016) que analiza el perfil de los profesores que asisten a congresos, concluyendo que la mayoría son profesoras (no catedráticas ni doctorandas) de una universidad pública de gran capital, que presentan trabajos con alta aplicación empírica, generalmente análisis de contenido descriptivos exploratorios y con resultados aplicables en el entorno privado, fundamentalmente en el área empresarial. En esta línea se sitúa el presente trabajo actualizando los datos sobre ponencias presentadas a los congresos de Comunicación.

\section{Contexto de investigación}

La investigación científica en España, y especialmente sobre Comunicación, se desarrolla principalmente en el entorno universitario. Es el personal docente e investigador quien tiene encomendada institucionalmente la tarea de ampliar el conocimiento científico. Así pues, las labores investigadoras conviven con las docentes, en una retroalimentación constante entre el descubrimiento y la difusión del conocimiento. 
Ahora bien, investigar en las ciencias de la Comunicación no es fácil, sobre todo desde la crisis económica de 2008 que hizo disminuir notablemente los fondos públicos para proyectos de investigación competitivos sin los cuales no se puede afrontar una investigación de cierta envergadura. Sucede además que cuando existen los fondos, los proyectos del área de Humanidades y Ciencias Sociales al que pertenece Ciencias de las Comunicación reciben cuantías hasta 4 veces inferiores de las que obtienen de media en el área de Ciencias de la Vida (EC3metrics, 2015). Las ayudas disminuyen al tiempo que se incrementan las presiones a los investigadores por una carrera académica altamente competitiva (Giménez-Toledo, 2016), a lo que no es ajeno el actual mecanismo que imponen las agencias de acreditación para conseguir consolidar y promocionar el status laboral (Goyanes, 2015). El sistema establecido, a menudo criticado por premiar más la cantidad que la calidad (Buela-Casal, 2002; Túñez-López, 2013), exige priorizar la investigación sobre la docencia por su mayor rédito en la consolidación laboral.

Esta relación entre investigación y docencia lleva a Bustos-González (2019) a analizar las diferencias entre "universidades de investigación", "universidades que hacen investigación" y "universidades docentes", en función de su producción científica anual, pues no todas las universidades tienen la misma productividad científica, señalando que no existe una correlación positiva entre desempeño investigador y tamaño institucional. Y si el tamaño de la universidad no es determinante, sí pueden influir otras características del centro, como la existencia de programas de doctorado, el mayor o menor incentivo a actividades y ayudas al profesorado o la existencia de grupos de investigación, elementos que De-Aguilera-Moyano (1998, p. 7) engloba en el "contexto institucional", junto con otros condicionantes del "contexto científico", refiriéndose a las corrientes y paradigmas dominantes, o a las "condiciones histórico-sociales" en las que se inscribe la práctica investigadora, puesto que la investigación está circunscrita al momento y lugar donde se realiza.

La influencia de estos contextos y características en la práctica de la investigación obligan a considerar estas variables en cualquier aproximación a la producción científica concreta (Izquierdo-Iranzo, 2019), a las que habría que añadir el contexto más inmediato, como la especialización, la comunidad o el "colegio invisible" que lo conforma, la agenda científica, los "call for papers"..., y aún las condiciones personales, familiares y económicas de quien investiga, que pueden facilitar o dificultar el trabajo investigador. De este modo se apunta cierta "desigualdad" de oportunidades de la que parten los investigadores por la mera pertenencia a un área del conocimiento u otro, o a una institución más prestigiosa y con más recursos e infraestructuras que otra e, incluso, a las características personales de los investigadores como la edad y la procedencia geográfica (González-Ramos et al., 2006, p. 205). Características a las que habría que añadir necesariamente el género, tal como constata la bibliografía científica (Jensen, 2005; Heidari et al., 2016; Caffarel-Serra; Ortega-Mohedano; Gaitán-Moya, 2018; Caffarel-Serra; Izquierdo-Iranzo; Núñez-Puente, 2018) y los informes de organismos oficiales, tanto nacionales (Unidad de Mujeres y Ciencia, 2016) como internacionales (Comisión Europea, 2016), que han señalado las barreras a la investigación en función del género.

Se añade a este escenario la dificultad a la hora de difundir los resultados de la investigación, toda vez que se objetiva la investigación realizada a través de publicaciones o ponencias presentadas en encuentros científicos. La exigencia de contar con un mayor número de aportaciones científicas para validar el currículum en un entorno tan competitivo como el actual ha ocasionado que se multipliquen las revistas científicas, por un lado, y los encuentros y congresos, de otro, en cada área de conocimiento. En este último caso, no sólo se advierte este fenómeno de concurrencia de citas científicas, también aumentan las comunicaciones presentadas y aceptadas en cada reunión. Esta dinámica, en la que no están ajenas las cuestiones económicas, dado que se cobra por acudir, perjudica su consideración, pues la inflación de comunicaciones cuestiona su calidad. Otros autores (Piñuel-Raigada; Lozano-Ascencio; García-Jiménez, 2011) añaden la selección basada en abstracts y escasamente justificada, la heterogeneidad de las mesas, el abultado número de firmantes por comunicación, la falta de especialización de los congresos y el escaso número de participantes senior frente a los de menor experiencia, como factores que perjudican la desigual calidad de los congresos.

Estas prácticas que desvirtúan los valores que deben inspirar la actividad científica reivindican el papel vigilante que debe tener la comunidad académica en su trabajo diario, visibilizándose a través, por ejemplo, de las asociaciones y colegios profesionales. En Comunicación, tras un intento fallido en los ochenta, se creó en 2007 la Asociación Española de Investigadores de la Comunicación $(A E-I C)$ siendo uno de sus objetivos

"difundir el conocimiento científico en Comunicación entre la sociedad civil a través de los grupos, congresos y las actividades promovidas por la entidad",

convocando al año siguiente en la Universidad de Santiago de Compostela su primer congreso, que congregó a más de medio millar de investigadores, y estableciendo una cita bianual que ha concentrado en los sucesivos encuentros (Málaga, 2010; Tarragona, 2012; Bilbao, 2014; Madrid, 2016 y Salamanca, 2018) ese "colegio invisible" de la Comunicación.

Las comunicaciones presentadas a los congresos no sólo difunden la actividad de los asociados, sino de todos los que investigan en el área, dado que no se exige ser socio. Además, existe cierta garantía de calidad de las comunicaciones presentadas por la existencia de un comité científico y la exigencia de presentar un paper para la publicación en actas, lo que requiere una mayor fundamentación teórica y metodológica. De esta manera, los congresos de la $A E-I C$ intentan paliar las carencias previamente mencionadas y es por lo que se han seleccionado para la presente investigación. 


\section{Metodología del estudio}

Este estudio se basa en datos primarios y empíricos, en cuanto que los datos se han obtenido de las propias comunicaciones presentadas a los congresos en un proceso de investigación realizado "exprofeso" aplicando un análisis de contenido. Para ello, se ha establecido un corpus de análisis conformado por todas las comunicaciones publicadas en los libros de actas de los congresos del $A E-I C$ por autores procedentes de universidades españolas. En total han sido analizadas 847 comunicaciones.

Una vez establecido el corpus y la unidad de análisis, se establecen las variables y categorías que van a permitir clasificar las comunicaciones y realizar los análisis posteriores. Teniendo en cuenta que el objetivo principal es conocer quién y qué se presenta en los congresos de la $A E-I C$, se han establecido dos grupos de variables:

- autoría, y

- contenido.

En el primer grupo se ha computado el género y la universidad del primer autor. Para operacionalizar el contenido, el proceso ha sido más complejo, puesto que no existe una única clasificación que cumpla con los objetivos de rigor y consenso exigibles en el contexto científico, además de que se perseguía una categorización que permitiera que todas las comunicaciones se pudieran clasificar de manera exhaustiva y excluyente. Por ello, dado que incluso la clasificación por áreas que propone la $A E-I C$ atiende a perspectivas teóricas, objetos de estudio, medios y métodos de investigación, se ha decidido distinguir al menos dos criterios de clasificación, atendiendo al tema u objeto del que trataba la comunicación, y al medio o soporte en que lo hacía, en la medida en que las contribuciones sobre fenómenos comunicativos suelen distinguir ambos aspectos. Así, por ejemplo, en la comunicación "Explorando el papel digital como plataforma para la difusión de contenidos periodísticos: resultados de una prueba piloto con Diario de Navarra" (R.S.) se puede distinguir el objeto de estudio, en este caso la incidencia de un nuevo dispositivo tecnológico como es el papel digital, aplicado a un medio, en este caso, el periódico navarro. De la comunicación "El consumo de contenido radiofónico en España en el nuevo ecosistema de medios digitales" (E.M.) se distingue también el objeto, en este caso el consumo, del medio referido, la radio.

Siguiendo este procedimiento, y codificando de una manera abierta, se ha llegado a una primera catalogación obteniendo un total de 32 categorías. Como el objetivo era realizar análisis estadísticos de frecuencias y cruces de las variables consideradas, se ha realizado una segunda catalogación para obtener un listado más depurado distinguiendo 11 aspectos o enfoques en los que se centraban las ponencias:

- Estudios históricos

- Rutinas profesionales

- Corporate (los medios como marca)

- Perspectiva crítica (estructura de los medios o las políticas públicas de comunicación)

- Contenidos

- Efectos

- Consumo y audiencia

- Participación ciudadana

- Nuevas tecnologías

- Enseñanza

- Investigación.

En lo que respecta a los medios, la catalogación ha sido más fácil puesto que se han diferenciado los formatos, tanto tradicional: prensa, TV, radio, publicidad, relaciones públicas..., como los medios online: internet, redes sociales o móviles. Ha habido ponencias que han tratado otros objetos, como agencias, comics, museos, música o fotografía, pero su escasa frecuencia ha recomendado agruparlos bajo la categoría de "otros". En el proceso de codificación se han cumplimentado las variables consideradas por cada una de las 847 ponencias presentadas y se han realizado análisis de frecuencias y cruces con los datos obtenidos.

\section{Resultados de la investigación y discusión}

El primer dato relevante de la investigación es la propia conformación del universo de estudio, 847 comunicaciones presentadas y aprobadas en los congresos celebrados por la $A E-I C$. Dejando a un lado el debate de la cantidad/calidad, lo cierto es que ha habido 847 autores, o equipos de autores, que han pensado, reflexionado, investigado, escrito y presentado una contribución científica en torno a fenómenos relacionados con la Comunicación, lo que consolida de alguna manera este campo de estudio.

Este número, cuanto menos notable, de comunicaciones guarda cierta correspondencia con el número de centros y universidades que ofrecen titulaciones de Comunicación, en la actualidad 68 centros (Civil-Serra, 2018), pues tal cantidad de universidades supone la concurrencia de numerosos profesores que necesitan presentar comunicaciones en congresos científicos para acreditar y competir por su carrera académica. 
Sin embargo, la presentación de ponencias en los congresos de la $A E-I C$ no ha respondido de manera directa a la pauta de crecimiento de los centros y de los profesores que trabajan en ellos. A mediados de los noventa sólo había unos 20 centros en España que ofrecieran titulaciones en Comunicación, una cifra que prácticamente se duplica a los diez años. De mediados de 2000 a la actualidad, período donde se suceden los congresos, los centros se vuelven a duplicar, hasta llegar a los 68 actuales. Pues bien, salvo el congreso inicial de 2008 en Santiago de Compostela donde se presentan 191 comunicaciones, en el que más, la $A E-I C$ sigue una pauta más bien restrictiva ya desde el siguiente congreso celebrado en Málaga en 2010, donde sólo se presentaron 107 ponencias, las mismas que en el último, celebrado en 2018.

Así pues, la cantidad de ponencias presentadas no responde a la demanda existente, suponiendo que se incremente a medida que se multiplica el público objetivo al que va dirigido, sino que puede responder a cuestiones más pragmáticas, como la crisis económica que incide en la disminución de las ayudas para viajes y congresos, y en los bolsillos de los congresistas, o en el lugar de celebración del congreso en cuestión. El espíritu descentralizador que inicialmente se propuso el $A E-I C$ al elegir la sede de los congresos (Rodríguez-Gómez, 2016) intentó minimizar el peso de las universidades centrales a favor de lo que en el último congreso sectorial de la $A E-I C$ se denominan "centros de la periferia", trasladando la sede a diferentes localidades de la geografía nacional. De este modo, no sólo se favorece que los investigadores de toda España conozcan otros centros, sino también que haya cierta deferencia con los investigadores locales. Y así fue en el primer congreso, los resultados de este estudio confirman que la reunión a la que más se han presentado los investigadores ga-

Las localizaciones geográficas protagonistas son Madrid y Cataluña, por ese orden, con 204 comunicaciones la primera y 163 la segunda, a lo que no es ajeno el protagonismo de sus universidades de referencia (Complutense y Autònoma)

llegos es a la de Santiago. También sucedió lo mismo en Bilbao, Madrid y Tarragona, donde hubo mayor afluencia de investigadores locales. Sin embargo, no sucedió lo mismo en Málaga o en Salamanca: de las 47 comunicaciones que presentó el profesorado de Málaga, sólo 8 lo hicieron en el congreso andaluz. Aún más significativo resulta lo que sucedió con los investigadores de la Universidad de Salamanca, que en el congreso auspiciado en su casa sólo participaron con 3 ponencias, frente a las 10 de Santiago o las 8 de Málaga. A pesar de estos ejemplos, en los que los investigadores locales desistieron de beneficiarse de una medida claramente descentralizadora, y a pesar también de la mejora de los transportes y la emergente participación digital que minimizará el peso de la distancia geográfica para asistir a los congresos, cabe admitir la existencia de cierta brecha en lo que a investigación en Comunicación se refiere entre unas universidades y otras según la zona geográfica en la que se ubiquen.

\subsection{Representatividad de las universidades y de las zonas geográficas de los ponentes}

Efectivamente, de las zonas de procedencia de los ponentes que se presentan a los congresos de la $A E-I C$ se puede decir que las localizaciones geográficas protagonistas son las comunidades que centralizan el poder institucional: Madrid y Cataluña, por ese orden, con 204 comunicaciones la primera y 163 la segunda, a lo que no es ajeno el protagonismo de sus universidades de referencia (Complutense y Autònoma) y la gran cantidad de universidades existentes en dichas comunidades: 12 centros en Madrid que ofrecen títulos de Comunicación, otros 12 en Cataluña, las comunidades que más universidades en Comunicación tienen y además pioneras en los estudios. También destaca Andalucía, pues supera el centenar de comunicaciones presentadas, no en vano es la Comunidad más extensa de la península, aunque no es la que más centros tiene, pues tiene sólo 6 instituciones, las mismas que Castilla y León. La concentración geográfica en Madrid, Cataluña y Andalucía coincide con la reportada en relación a la actividad científica de tesis doctorales y proyectos de investigación por Caffarel-Serra, Ortega-Mohedano y Gaitán-Moya (2018).

En número de ponencias presentadas sigue la Comunidad Valenciana, que concentra 9 centros que imparten Comunicación, con 89 ponencias. Otras comunidades autónomas también contribuyen de manera notable: Castilla y León (75), País Vasco (62); Galicia (58); Murcia (20); y Navarra (19). Cifras que contrastan con la aportación minoritaria de Comunidades Autónomas con cierta extensión, por ejemplo, Castilla La Mancha, que sólo aporta 8 comunicaciones, las mismas que Extremadura o las 9 de Aragón. En relación a las zonas más y menos productivas específicamente en congresos también encontramos sintonía con los resultados expuestos por Rodríguez-Gómez (2016), este autor indica como las más fructíferas: Cataluña, Madrid, Andalucía, País Vasco y Castilla y León, y como las menos: Aragón, Canarias, La Rioja, Castilla La Mancha y Baleares. Es decir, que se coincide en las comunidades que mayor actividad tienen, salvo en la Comunidad Valenciana; y en Aragón y Castilla La Mancha como comunidades que menos actividad tienen. Hay que tener en cuenta que el presente estudio cubre 4 años más, es decir, dos ediciones más de congresos que el estudio con el que se compara. Estos resultados que inciden en estas comunidades interiores con menor representatividad pueden responder a que son comunidades que concentran pocos centros que imparten titulaciones en Comunicación: 2 en Castilla La Mancha y en Aragón, 1 en Extremadura. Aunque el número de centros no determina la cantidad de profesores que envían comunicaciones, porque Navarra sólo tiene un centro y se sitúa en un nivel bastante notable de visibilidad, aunque ese centro es de los más antiguos en impartir docencia de Comunicación. Así pues, la visibilidad de las regiones en los congresos de la $A E-I C$ responde a la confluencia de distintos factores, fundamentalmente relacionados con los centros universitarios que albergan. 
En España hay contabilizados 49 centros, pero no todos ellos están representados en los congresos de la $A E-I C$, pues hay 18 que imparten titulaciones de Comunicación que nunca han enviado a algún profesor. Dentro de las universidades representadas, no todas presentan el mismo número de investigadores. Haciendo una primera discriminación por la titularidad, el peso de ponentes procedentes de universidades públicas es mayoritario (un 86\%). Teniendo en cuenta que prácticamente la mitad de los 68 centros universitarios que imparten titulaciones en Comunicación son públicos, se puede concluir que los centros que no están representados son eminentemente los privados, que se dedican, siguiendo la terminología de Bustos-González (2019) a ser centros de enseñanza más que de investigación. Entre los privados que presentan comunicaciones, el peso más notable se lo lleva el CEU, tanto el centro de Valencia como el de Madrid, con cerca de 20 comunicaciones presentadas desde cada sede, igual que la Universidad Internacional de La Rioja, el otro centro privado que más comunicaciones ha presentado. El protagonismo de la universidad pública frente a la privada en tareas de investigación es así mismo observado por otros autores (Rodríguez-Gómez, 2016; Caffarel-Serra; Ortega-Mohedano; Gaitán-Moya, 2017).

La universidad pública que más comunicaciones ha presentado es la Complutense de Madrid, seguida de la Autònoma de Barcelona. A continuación se sitúan la Universidad Rey Juan Carlos y la Universidad del País Vasco, siendo estas cuatro las únicas que superan las 50 comunicaciones presentadas en los 6 congresos. Por comparar resultados, en un estudio realizado en revistas, de los 256 artículos analizados

"la universidad con mayor presencia es la Autònoma de Barcelona, con un 12,4\% de los autores y con un $8,7 \%$ encontramos la categoría de instituciones no universitarias. El tercer puesto lo ocupa la Universidad Complutense de Madrid con un 8,2\%" (Castillo-Esparcia; Carretón-Ballester, 2010, p. 310).

Como se puede ver en la tabla 1 , existe otro conjunto de universidades con representación notoria en los congresos de la $A E-I C$, entre las que destacan la de Málaga y la de Sevilla. Universidades con cifras prolíficas, pero ya por debajo de la cuarentena de participaciones son: la Carlos III de Madrid, la Jaume I, la de Salamanca, la de VaIladolid y la Pompeu Fabra. Cerrando el ranking de las que tienen más de 25 se sitúa la de Santiago de Compostela. Tratando de explicar el ranking apuntado, se pueden señalar ciertas razones que inciden en la mayor o menor representación de los centros, como la ubicación geográfica y la mayor disposición y facilidad para viajar, pero también si son zonas privilegiadas para tener recursos o ayudas tanto para investigar como para asistir a congresos. También influye obviamente el centro en cuestión, pues ya sea por el tamaño, Tabla 1. Ranking de universidades por número de comunicaciones presentadas a los congresos de la $A E-I C$ y género mayoritario de los docentes

\begin{tabular}{|l|c|c|}
\hline Universidad & No de comunicaciones & Género mayoritario \\
\hline Complutense & 73 & Masculino \\
\hline Autònoma de Barcelona & 67 & Femenino \\
\hline Rey Juan Carlos & 61 & Masculino \\
\hline País Vasco & 53 & Femenino \\
\hline Málaga & 47 & Femenino \\
\hline Sevilla & 46 & Femenino \\
\hline Carlos III & 36 & Masculino \\
\hline JaumeI & 35 & Masculino \\
\hline Salamanca & 32 & Masculino \\
\hline Valladolid & 30 & Masculino \\
\hline Pompeu Fabra & 30 & Masculino \\
\hline Santiago & 29 & Masculino \\
\hline
\end{tabular}

por su importancia geopolítica o por su valor diferencial, pueden facilitar más o menos la investigación y su difusión, con ayudas a congresos o apoyo a grupos de investigación. De hecho, los centros geográficos más representados son los que tienen más grupos de investigación en Comunicación: Madrid 39, Cataluña 35, Andalucía 33 o Valencia 26 (Tur-Viñes; Núñez-Gómez, 2018). Pero también hay que apuntar otras razones que responden a lo que se podría denominar el momento vital que viva la universidad, que ya sea por tradición o por la configuración de su cuadro docente, tienen más predisposición para presentar comunicaciones y asistir a congresos que otras con historias o momentos vitales distintos.

\subsection{Ponentes: investigadoras e investigadores}

Los datos del análisis de las comunicaciones presentadas a los congresos de la $A E-I C$ confirman que existe paridad de género en la investigación en Comunicación en España. Es más, en el conjunto de las comunicaciones presentadas, las 434 mujeres (51,3\%) superan a los varones $413(48,7 \%)$. Este resultado presenta una razonable coincidencia con los de investigaciones equivalentes: $54 \%$ de participación femenina y $46 \%$ masculina en congresos de Comunicación (Rodríguez-Gómez, 2016) y se asemeja en proporción y nivel con los hallazgos de objetos comparables (Izquierdo-Iranzo; Gaitán-Moya; Caffarel-Serra, 2020), donde se reporta un ligero mayor peso femenino en la autoría de las tesis doctorales de Comunicación (primer nivel de la investigación equiparable al de las ponencias), pero se constata brecha de género a favor de los hombres en niveles superiores de investigación, en concreto en la dirección de proyectos I+D.

Si bien en el conjunto de los congresos las mujeres superan a los varones, no sucede así en todas las ediciones. En el primero, en Santiago, hay más hombres que mujeres, de las 191 comunicaciones, 107 son presentadas por varones y 84 por mujeres, pauta que ya en el segundo congreso, en Málaga, dos años después, cambia notablemente, invirtiéndose el orden, y las mujeres ya superan a los varones, lo cual se repetirá a partir de entonces, pues en ninguno de los siguientes congresos los hombres superan a las mujeres, es más, excepto en el de Madrid donde el peso de unos y otras se equilibra a un $50 \%$, en los demás siempre las mujeres superan a los hombres en la presentación de las comunicaciones. La 
progresión temporal descrita a favor de la participación femenina en los congresos tiene sentido en el marco global de avance de los derechos de las mujeres ligado al paso del tiempo, que se refleja tanto a nivel jurídico (Ley $3 / 2007$ de 22 de marzo por la igualdad efectiva entre hombres y mujeres) como en el social con la llamada "cuarta ola del feminismo".

Tabla 2. Porcentaje de ponentes en función del género

\begin{tabular}{|l|c|c|c|c|c|c|}
\cline { 2 - 7 } \multicolumn{1}{c|}{} & Total & Santiago & Málaga & Tarragona & Bilbao & Madrid \\
\hline Mujer & 51,2 & 44 & 59 & 52 & 55 & 50 \\
\hline Hombre & 48,8 & 56 & 41 & 48 & 45 & 50 \\
\hline
\end{tabular}

Sin embargo, el dato global de la ligera superioridad de participación femenina en los congresos no se reproduce cuando se analizan las universidades individualmente, en este caso encontramos un panorama cambiante, tal como se puede comprobar en la tabla 1.

Sí hay algunas universidades importantes donde predominan las mujeres, como la Universitat Autònoma de Barcelona, la del País Vasco o la Oberta de Catalunya, y otras en las que el peso de un género y otro se equilibra, como es el caso de la Universidad de Málaga y la de Sevilla. Sin embargo, en las universidades madrileñas: la Complutense, la Rey Juan Carlos y la Carlos III; en la Universidad de Santiago, la Jaume I y la Pompeu Fabra, la presentación de comunicaciones por parte de investigadores varones supera las que presentan sus compañeras.

Si analizamos la distribución del género agrupando las universidades por comunidades autónomas se revela que el mayor peso de las investigadoras o, al menos, en una proporción paritaria, se logra en todas las comunidades autónomas excepto en Madrid, Cataluña y Aragón, comunidades donde los investigadores varones presentaron más comunicaciones que las mujeres. El caso de Aragón no resulta significativo dada la escasa relevancia de su aportación, aunque lo cierto es que de 8 comunicaciones que se presentaron sólo una fue de una mujer. Más relevante es lo de Madrid y Cataluña: tanto en una comunidad como en la otra, los varones representan el $51,5 \%$ de las ponencias presentadas.

Estos datos pueden compararse con estudios que analizan también la producción científica desde la perspectiva de género, y si en el presente estudio de comunicaciones presentadas se muestra cierto desequilibrio a favor de ellos en la autoría de ponencias de las universidades madrileñas y algunas de las universidades de: Galicia, Comunidad Valenciana, Castilla León y Cataluña, en el estudio de Izquierdo-Iranzo, Gaitán-Moya y Caffarel-Serra, que analizan las tesis doctorales se concluye que

“Murcia, Canarias, Andalucía y Cataluña siguen presentando una mayoría de tesis firmadas por varones, mientras que la autoría de las mujeres se impone en Madrid, Galicia, Comunidad Valenciana, Castilla-León, País Vasco, Navarra, Extremadura y Baleares" (Izquierdo-Iranzo; Gaitán-Moya; Caffarel-Serra, 2020).

Es decir, únicamente es en la Universidad del País Vasco donde ambas investigaciones encuentran mayoría de autoría femenina, allí, tanto las tesis como las comunicaciones son, sobre todo, presentadas por ellas. También se coincide en mayor brecha de género en las universidades madrileñas: si en el presente estudio se comprueba un claro desequilibro a favor de los investigadores en cuanto a la producción de ponencias, en Izquierdo-Iranzo, Gaitán-Moya y Caffarel-Serra (2020) se observa que los centros de Madrid encabezan el índice de desigualdad en la dirección de proyectos I+D, pero en IP (investigador principal) también el País Vasco muestra esa diferencia, en consonancia con el techo de cristal que también existe en la investigación en Comunicación, mayor desigualdad cuanta mayor importancia tiene la investigación.

Que los resultados sobre el desequilibrio de género en la producción científica varíen por el tipo de producción científica que se considere, que cambie también según las regiones y universidades nos habla del cambiante y evolutivo momento en el que se encuentra el papel de la mujer en la sociedad y, por extensión, el de su protagonismo en la ciencia.

\subsection{Temas o problemas de investigación}

Las ponencias que se presentan a los congresos que ha celebrado la $A E-I C$ son muy distintas, lo cual manifiesta la creatividad académica. No obstante, a pesar de esta gran variedad, es posible encontrar alguna tendencia en los intereses y asuntos que más preocupan a la comunidad académica española, tratando de agrupar en grandes categorías los asuntos de los que tratan las comunicaciones. Los datos del estudio indican que hay algunos objetos de estudio recurrentes en la investigación en Comunicación y, tal como muestra el gráfico 1, uno de ellos son los contenidos mediáticos, y otros que se tratan de manera más puntual.

Uno de estos enfoques minoritarios ha sido el que aborda la perspectiva histórica de los fenómenos comunicativos, pues solamente un $2 \%$ de las comunicaciones tratan esas cuestiones, como “El documental en Televisión Española. Los orígenes del género" (M.N.). Este dato es destacable pues el estudio histórico es un enfoque tradicional en las teorías de la Comunicación. 
También minoritaria ha sido la pauta de abordar la Comunicación en contextos educativos: sólo el 3,4\%, ya sea procesos de alfabetización mediática o como objeto de enseñanza. Esta escasez llama la atención porque, al fin y al cabo, los ponentes son en su mayoría docentes de las titulaciones universitarias. Más interés recibe la investigación, y es que hay un elenco interesante de comunicaciones, un 7,6\% del total, que se centran en la meta-investigación de la Comunicación. Son exactamente 65 ponencias que suelen plantear propuestas

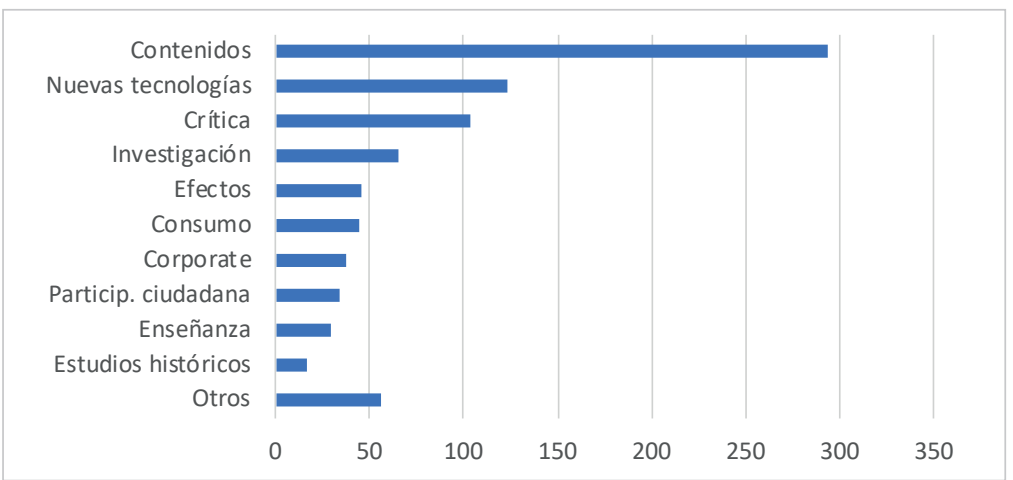

Gráfico 1. Temas principales de las comunicaciones presentadas a los congresos de la $A E-I C$ metodológicas para el estudio de fenómenos comunicativos concretos. De hecho, muchas de las comunicaciones presentan los planteamientos metodológicos de proyectos más ambiciosos que suelen corresponder a proyectos competitivos.

Analizando los temas desde la perspectiva del fenómeno comunicativo según el esquema tradicional, son frecuentes las investigaciones enfocadas al emisor, bien sea analizando las prácticas profesionales (5\%) como "Estrés en publicitarios españoles. Un estudio exploratorio de causas y consecuencias de la tensión en las agencias" (M.M.LL.); o su ámbito más corporativo y comercial (4\%) como "La responsabilidad social corporativa en los medios de comunicación: el caso de El país" (F.C.G.). Observamos un alto porcentaje de ponencias, un $12 \%$, que analizan la Comunicación desde la perspectiva crítica, bien la estructura mediática (9\%) o la incidencia de las políticas públicas en el funcionamiento de sistemas comunicativos concretos (3\%).

También encontramos ponencias sobre los usuarios, bien sea analizando su consumo (5\%) o algún aspecto de los efectos mediáticos (6\%). De manera especial se ha considerado la participación ciudadana por ser el objeto principal de 34 comunicaciones presentadas en la $A E-I C$, lo que representa el $4 \%$ del total. Son los años de eclosión de las redes sociales y del 11-M. Una de ellas es "Ciudadanos, consumidores, colaboradores y activistas: estrategias de participación de la audiencia en la televisión multiplataforma" (J.A.G.A.). Especial mención tienen las comunicaciones que se refieren al efecto de la digitalización, las tecnologías o las redes, con un 14,5\% del total, como "La nueva televisión local en Andalucía: Internet como ámbito de desarrollo para las emisoras de proximidad digitales" (C.M.) o "Modelo de desarrollo de una plataforma de IPTV de producción infantil" (A.S.). Es hasta cierto punto lógica esta focalización dado que los congresos son foros donde se presentan las investigaciones o reflexiones más actuales de la Academia, y en este siglo el impacto de la convergencia digital es lo que más ha transformado el sistema comunicativo y la sociedad en su conjunto.

Aun así, no es la disrupción tecnológica el principal elemento de investigación y preocupación, sino los contenidos mediáticos: un 34,5\% de las ponencias presentadas se refieren a ellos: 293 comunicaciones de las 847 analizadas, entre las que se cuentan "La comunicación digital de las marcas de moda: un análisis comparativo entre Facebook y Twitter" (M.N.) o "Del Mitch a Fukushima pasando por Haití: las emergencias ambientales en las redes sociales de las ONGD" (V.S.). Dentro de esta categoría general de contenidos, es posible distinguir a su vez diferentes temas de análisis, ciertos campos de interés en los que han redundado más comunicaciones, y de entre ellos, destaca el político, ya sean las campañas electorales, los debates o la construcción de los líderes. La comunicación política ha sido el tema principal de 63 ponencias (7,4\%). Otros temas especializados han sido salud $(2,7 \%)$, crisis $(2 \%)$, cultura $(1,9 \%)$, medioambiente $(0,8 \%)$, ciencia $(0,6 \%)$ y deportes $(0,4 \%)$.

\subsection{El medio investigado}

Se ha analizado de manera diferenciada el soporte principal de investigación (gráfico 2), aunque no todas las ponencias se referían a un tipo de medio específico ya que 134 ponencias trataban la Comunicación de manera genérica y 31 se referían a otros ámbitos distintos de la Comunicación, como el teatro o la música. De las restantes, las que se referían a medios tradicionales (561) se han distinguido de la referidas a los online (121). Un análisis más detallado señala que el medio principal de referencia es el audiovisual, con 274 ponencias, agrupando el audiovisual genérico (59 comunicaciones), y otros medios audiovisuales específicos. De estos últimos, se prefiere la televisión (99 comunicaciones). Aunque es posible que este papel protagónico de la televisión como medio de referencia cambie dado el creciente peso del mundo digital. De hecho, en 2019 por primera vez la inversión publicitaria en medios digitales superó a la de la televisión convencional (Infoadex, 2020). También tiene importancia la radio (57 ponencias). De manera minoritaria se ha tratado el cine (31 comunicaciones), las series (18) y los videojuegos (10).

Después de los audiovisuales, el medio más tratado en las comunicaciones es la prensa, dada la mayor disponibilidad del objeto material de estudio, hecho que coincide con otros estudios que analizan los medios principales que refieren los artículos de investigación en revistas científicas, como el de Castillo-Esparcia y Carretón-Ballester (2010) donde el protagonismo es del periodismo seguido de la televisión y, de lejos, la publicidad, las relaciones públicas e internet y las nuevas tecnologías.

La concentración de la actividad científica en Madrid, Cataluña y en el litoral permite hablar de una España vacía o vaciada en la investigación en Comunicación 
Así sucede también en el presente estudio, donde se han distinguido las ponencias sobre publicidad de las de relaciones públicas. "La aparición del cuerpo humano en la publicidad" (A.CH.), es una de las 57 ponencias computadas que se referían a la publicidad, mientras que "La comunicación en los organismos nacionales del fútbol" (G.L.) lo es de las 47 comunicaciones presentadas dentro de las relaciones públicas.

El análisis se completa con las ponencias que han estudiado los nuevos medios, distinguiendo las que trataban las redes sociales (46 ponencias), como "El Real Madrid C.F. y sus patrocinadores en la red social Facebook" (J.M.P.); las 10 ponencias que investigaban la comunicación a través del móvil, como "Uso y percepciones de dispositivos móviles" (K.O.); y las 65 comunicaciones que analizaban Internet, como "Nuevos modelos de participación y empoderamiento ciudadano a través de internet" (M.D.C.) presentada en el congreso de Madrid.

La diferencia que aún existe entre el estudio de los medios tradicionales y los nuevos medios se muestra en consonancia con el obtenido en el proyecto I+D MapCom:

"sorprende el porcentaje tan minoritario 22,96\% de investigaciones sobre objetos de estudio comunicacionales online frente al 66,90\% de esas investigaciones sobre objetos presenciales" (Caffarel-Serra; Ortega-Mohedano; Gaitán-Moya, 2018, p. 224).

Hay que tener en cuenta que el mundo digital no tenía antes la importancia actual y no existía como referente ni en la formación ni en la vida de la mayoría de los investigadores que hoy en día estudian los fenómenos comunicativos.

\section{Conclusiones}

En el camino para hacer de la Comunicación un campo consolidado de estudio y un área con cierta entidad en España ha contribuido notablemente la Asociación Española de Investigadores de la Comunicación, no sólo por constituir una referencia, sino también por formalizar una cita bienal de encuentro entre los investigadores y de difusión, cuando no de incentivo, de las investigaciones y reflexiones en torno a este fenómeno. Pues bien, se ha constatado a través de este estudio la dimensión cuantitativa de esta aportación: 847 comunicaciones. Esta cifra permite confirmar una notable producción de investigación en Comunicación en nuestro país, en consonancia con la gran cantidad de investigadores existentes en esta área de conocimiento, consecuencia a su vez de la multiplicación de centros que imparten titulaciones de Comunicación, cerca de 70 actualmente en nuestro país. Hay que señalar que cerca de la mitad son centros privados, pero esa relación no se manifiesta de la misma manera en las comunicaciones presentadas, pues los profesores de universidades privadas no participan apenas en los congresos de la $A E-I C$, evidenciando que las universidades privadas son más bien centros de enseñanza y son las públicas las que concentran la investigación.

El estudio empírico realizado sobre las comunicaciones publicadas en los congresos de la $A E-I C$ permite profundizar en algunas características de esa producción científica, sobre quiénes la hacen, desde dónde o sobre qué. Aunque las comunicaciones a estos congresos no reflejen toda la investigación sobre Comunicación en España, puesto que no es investigación todo lo que se presenta ni se presenta allí toda la investigación que se hace, sí pueden ser indicativas del trabajo que realizan los investigadores españoles, porque estos congresos constituyen el lugar de encuentro de la comunidad científica en Comunicación y, por ende, constituyen uno de los escaparates de su producción científica. Pues bien, lo primero que se puede decir es que existe cierta brecha geográfica en la investigación española en Comunicación que concentra la actividad científica en ciertos lugares privilegiados: sobre todo las comunidades principales, Madrid y Cataluña, y el litoral mediterráneo, Andalucía y Valencia, en detrimento de las zonas geográficas centrales, de modo que también se puede hablar de una España vacía o vaciada en la investigación en Comunicación.

Si se habla de investigación también es importante destacar la universidad de procedencia, dado que la investigación en España se realiza sobre todo en las universidades. Además, siendo como es el encuentro bienal de la comunidad científica, la cantidad de investigadores por universidad es un indicador de la visibilidad de las facultades en el escaparate nacional y de su contribución al campo de estudio. La universidad con mayor visibilidad es la Complutense de Madrid, seguida de la Autònoma de Barcelona, por ser de mayor tamaño, pioneras y de referencia en los estudios de Comunicación. También tienen cierto protagonismo la Universidad Rey Juan Carlos y la Universidad del País Vasco, al menos por el número de ponencias presentadas, la primera por el elevado número de docentes con que cuenta, la mayoría haciendo su carrera académica, y la segunda por tradición e historia. 
Y si interesa conocer la universidad donde trabajan los ponentes que presentan comunicaciones, también amerita conocer su género: Siguiendo el indicador de las comunicaciones presentadas en los Congresos de la $A E-I C$ se puede decir que se ha roto la barrera del género, son más mujeres que hombres quienes presentan comunicaciones. Es posible hablar de cierta evolución natural hacia la paridad según se va avanzando en niveles superiores de la carrera académica: empezando por las aulas, donde las mujeres ya son mayoría y constatando ya la paridad en el nivel doctoral y en las presentaciones a congresos, como se ha puesto de manifiesto en esta investigación. Es previsible su progresión a medida que pasa el tiempo en los niveles superiores, alcanzando la paridad primero en la firma de artículos científicos y después en los escalafones superiores del rango académico, consiguiendo en un futuro romper el techo de cristal que evidencia el número tan escaso de catedráticas de Comunicación o de investigadores principales de los proyectos.

Esa paridad en la presentación de comunicaciones no pasa en todas las universidades. El que haya una diferencia entre investigadores e investigadoras procedentes de un centro obedece a diversos factores, entre ellos, puede indicar una diferencia de género en el claustro o en los programas de doctorado. Sea como fuere, lo que sí dice la investigación realizada es que, aunque algunas universidades con cierta visibilidad en los congresos de la $A E-I C$, como la Autònoma de Barcelona o la del País Vasco, envían a más mujeres a presentar comunicaciones, no sucede lo mismo en la Complutense, la Carlos III ni en la Rey Juan Carlos. En estas tres universidades públicas madrileñas, que no envían precisamente a pocos docentes, son más los profesores que las profesoras. Pero no es Madrid la única comunidad autónoma donde predominan los varones, también pasa en Cataluña, y es significativo que en las dos comunidades políticamente significativas sigan predominando los varones. En todas las demás comunidades, excepto en Aragón, las mujeres representan el mismo peso que los varones, o más. A veces las únicas que asisten, como es el caso de Canarias o de Extremadura.

Y si interesa quiénes presentan comunicaciones, también interesa sobre qué, porque da cuenta de las inquietudes científicas de los investigadores en este campo. Una de las primeras conclusiones que se desprenden del estudio es la necesidad de contar con una catalogación exhaustiva y compartida de los temas principales de estudio, cuya inexistencia da cuenta de la complejidad que supone tal ejercicio taxonómico. A pesar de ello, se ha elaborado con una clasificación, a partir de las propias ponencias presentadas, que distingue el tema del que trata y el medio donde se estudia. Por lo que respecta a los temas, y siguiendo la vieja proclama de

121 ponencias tratan de manera principal los nuevos medios: internet, las redes o el móvil

McLuhan, podemos decir que lo que prima es el mensaje. A los investigadores les preocupan los contenidos, y a tenor del soporte principal de estudio, se investiga principalmente en prensa. Los contenidos pueden ser variados, y en una clasificación por especialidad periodística, el campo que más seduce es la política. Preocupa, en ese sentido, si la prensa sigue ejerciendo de cuarto poder, por eso se investiga su papel de vigía.

Pero a esta preocupación se suman otras, derivadas de la transformación digital. La Comunicación ha cambiado, y esos cambios forman parte natural de la agenda investigadora del área. Hay numerosas comunicaciones que abordan los nuevos medios (redes sociales, móviles...) o cómo estos cambios están afectando a la comunicación tradicional, especialmente la audiovisual. De hecho, es el audiovisual el medio más tratado por las ponencias de los congresos, considerándolo ya como un medio propio, dada la convergencia multimedia existente, o analizando los soportes convencionales: radio, cine, televisión, o nuevas expresiones del audiovisual actual: los videojuegos o las series. Efectivamente esa tendencia al predominio audiovisual que se percibe en el fenómeno comunicativo también se percibe, de manera correlativa, en la investigación sobre Comunicación.

Aun hay otra línea de investigación que goza de cierto asentamiento entre las comunicaciones presentadas en la $A E-$ $I C$, no en vano hay una sección específica sobre el tema: los estudios críticos que analizan la estructura mediática y la economía política de la Comunicación, protagonismo que deriva de la importancia de investigar a quienes controlan la comunicación, una configuración que cambia cada vez más deprisa, que convierte a las empresas en entidades cada vez más grandes y más oscuras, oligopolios de la Comunicación que la Academia trata de conocer sin terminar de lograrlo.

Estas son las principales inquietudes científicas que han ocupado a los investigadores españoles en los últimos 12 años. Han sido, además, unos años en los que ha cambiado notablemente el fenómeno de la Comunicación y la investigación ha respondido a ese reto a pesar de la crisis económica que no sólo mermó los fondos para investigar, también para asistir a los encuentros científicos donde se comparten esas inquietudes. A pesar de ello, el músculo investigador español ha sorteado el temporal y la asistencia a los congresos de la Asociación Española de Investigadores de la Comunicación ha sido masiva, en los 6 congresos que llevan celebrándose hasta ahora. En todos se ha superado el centenar de comunicaciones seleccionadas y publicadas en actas, sumando cerca de 850 , que permiten diagnosticar la buena salud del sector investigador en nuestro país.

\section{Referencias}

Aalbers, Manuel B. (2004). "Creative destruction through the Anglo-American hegemony: a non-Anglo-American view on publications, referees and language". Area, v. 36, n. 3, pp. 319-322.

Almiron, Núria; Reig, Ramón (2007). "The communications research in Spain: The political economy epistemological approach". American communication journal, v. 9, n. 2.

https://idus.us.es/handle/11441/28229 
Arcila-Calderón, Carlos; Calderín-Cruz, Mabel; Sánchez-Holgado, Patricia (2019). “Adopción de redes sociales por revistas científicas de ciencias sociales". El profesional de la información, v. 28, n. 1, e280105.

https://doi.org/10.3145/epi.2019.ene.05

Baladrón-Pazos, Antonio J.; Correyero-Ruiz, Beatriz; Manchado-Pérez, Benjamín (2014). "Three decades of advertising research in Spain: Analysis of scientific communication journals (1980-2013)". Communication \& society, v. 27 , n. 4, pp. $49-71$. https://doi.org/10.15581/003.27.4.49-71

Banfi, Antonio; Franzini, Elio; Galimberti, Paola (2014). Non sparate sull'umanista. La sfida della valutazione. Milano: Guerini e Associati. ISBN: 9788862505482

Barker, Katharine (2007). "The UK Research assessment exercise: the evolution of a national research evaluation system". Research evaluation, v. 16, n. 1, pp. 3-12.

https://doi.org/10.3152/095820207X190674

Barranquero-Carretero, Alejandro; Limón-Serrano, Nieves (2017). “Objetos y métodos dominantes en Comunicación para el desarrollo y el cambio social en las tesis y proyectos de investigación en España (2007-2013)". Revista latina de comunicación social, n. 72, pp. 1-25.

https://doi.org/10.4185/RLCS-2017-1151

Braun, Tibor; Dióspatonyi, Ildiko (2005). “World flash on basic research”. Sicentometrics, v. 62, pp. $297-319$. https://doi.org/10.1007/s11192-005-0023-7

Buela-Casal, Gualberto (2002). "Evaluación de la investigación científica: el criterio de la mayoría, el factor de impacto, el factor prestigio y los diez mandamientos para incrementar las citas". Análisis y modificación de la conducta, v. $28, \mathrm{n}$. 119, pp. 455-475.

https://dialnet.unirioja.es/servlet/articulo?codigo $=272362$

Bustos-González, Atilio (2019). "Tránsito de universidad docente a universidad de investigación. ¿Un problema de información académica, de taxonomías o de rankings universitarios?”. El profesional de la información, v. 28 , n. 4, e280422. https://doi.org/10.3145/epi.2019.jul.22

Cáceres, María-Dolores; Caffarel-Serra, Carmen (1993). “La investigación sobre Comunicación en España. Un balance cualitativo". Telos, n. 32, pp. 109-124.

https://telos.fundaciontelefonica.com/archivo/numero032/un-balance-cualitativo

Caffarel-Serra, Carmen; Domínguez, Milagros; Romano, Vicente (1989). “El estado de la investigación de Comunicación en España (1978-1987)". Cinco. Cuadernos de investigación en comunicación, n. 3, pp. 45-57.

Caffarel-Serra, Carmen; Izquierdo-Iranzo, Patricia; Núñez-Puente, Sonia (2018). “¿Cómo investiga la mujer cuando investiga sobre la mujer en Comunicación?". En: Martínez-Rodrigo, Estrella (coord.). Propuestas de investigación en áreas de vanguardia. Madrid: Tecnos, pp. 73-86. ISBN: 9788430973873

Caffarel-Serra, Carmen; Ortega-Mohedano, Félix; Gaitán-Moya, Juan-Antonio (2017). “Investigación en Comunicación en la universidad española en el período 2007-2014". El profesional de la información, v. 26, n. 2, pp. 218-227.

https://doi.org/10.3145/epi.2017.mar.08

Caffarel-Serra, Carmen; Ortega-Mohedano, Félix; Gaitán-Moya, Juan-Antonio (2018). “La investigación en Comunicación en España. Debilidades, amenazas, fortalezas y oportunidades”. Comunicar, n. 56, pp. 61-70. https://doi.org/10.3916/C56-2018-06

Casado, Miguel-Ángel; Fernández-Quijada, David (2013). “El estado de la investigación española en políticas de la Comunicación: una revisión bibliométrica (2002-2011)". Trípodos, n. 32, pp. 113-132.

https://core.ac.uk/download/pdf/39028822.pdf

Castillo-Esparcia, Antonio; Carretón-Ballester, María-Carmen (2010). "Investigación en Comunicación. Estudio bibliométrico de las revistas de Comunicación en España". Comunicación \& society, v. 23, n. 2, pp. $289-327$.

https://revistas.unav.edu/index.php/communication-and-society/article/view/36234

Castillo-Esparcia, Antonio; Xifra, Jordi (2006). "Investigación bibliométrica de las tesis doctorales españolas sobre relaciones públicas (1965-2005)”. Anàlisi. Quaderns de comunicació i cultura, n. 34, pp. 111-124. https://www.raco.cat/index.php/Analisi/article/view/55449

Civil-Serra, Marta (2018). "Los estudios universitarios de Comunicación en España (2017). Análisis de la situación actual para un debate abierto sobre su futuro estratégico". En: Postigo-Gómez, Inmaculada; Recoder-Sellarés, María-José. Los y las tics en los estudios de Comunicación. Asociación Española de Universidades con Titulaciones de Información y Comunicación, ATIC, pp. 15-83. ISBN: 9788409019731

Comisión Europea (2016). She figures (2015). Luxembourg: Publications Office of the European Union. https://ec.europa.eu/research/swafs/pdf/pub_gender_equality/she_figures_2015-final.pdf 
Costa-Sánchez, Carmen (2017). "Análisis de la productividad y visibilidad en Scopus de los investigadores españoles en Comunicación". Observatorio (OBS*), v. 11, n. 3, pp. 1-15.

https://doi.org/10.15847/obsOBS11320171030

De-Aguilera-Moyano, Miguel (1998). “La investigación sobre Comunicación en España: una visión panorámica”. Comunicación y cultura, n. 4, pp. 5-12.

https://dialnet.unirioja.es/servlet/articulo?codigo=2901273

De-Moragas, Miquel (1988). “Los estudios sobre Comunicación y nuevas tecnologías en España: indicaciones sobre sus antecedentes y estado actual”. Cinco. Cuadernos de investigación en Comunicación, n. 1, pp. 11-19.

Díaz-Nosty, Bernardo; De-Frutos-García, Ruth (coords.) (2016). Rumbos de la investigación en España sobre Comunicación. Málaga: eBook. ISBN: 9788460870531

EC3metrics (2015). Análisis de los resultados de la Convocatoria de proyectos I+D+l del Plan Nacional de Investigación. Granada: EC3metrics.

https://ec3metrics.com/2015/06

Escribá-Sales, Eudald; Cortiñas, Sergi (2013). "La internacionalización y las coautorías en las principales revistas científicas de Comunicación en España”. Comunicar, v. 21, n. 41, pp. 35-44.

https://doi.org/10.3916/C41-2013-03

Fernández-Quijada, David; Masip, Pere (2013). “Tres décadas de investigación española en Comunicación: hacia la mayoría de edad". Comunicar, n. 41, pp. 15-24.

https://doi.org/10.3916/C41-2013-01

Giménez-Toledo, Elea (2016). Malestar. Los investigadores ante su evaluación. Madrid: Iberoamericana. ISBN: 97884 84898184

Giménez-Toledo, Elea; Tejada-Artigas, Carlos (2012). "Valoración de editoriales especializadas en Comunicación, biblioteconomía y documentación: encuesta a profesores e investigadores". El profesional de la información, v. 21, n. 1, pp. $50-62$. http://doi.org/10.3145/epi.2012.ene.07

González-Ramos, Ana; González-de-la-Fe, Teresa; Fernández-Palacín, Fernando; Muñoz-Márquez, Manuel (2006). “Idoneidad de los indicadores de calidad de la producción científica y de la investigación". Política y sociedad, v. 43, n. 2, pp. 199-213.

https://revistas.ucm.es/index.php/POSO/article/view/POSO0606220199A

Gorraiz, Juan (2018). "Los mil y un reflejos de las publicaciones en el laberinto de espejos de las nuevas métricas". El profesional de la información, v. 27, n. 2, pp. 231-236.

https://doi.org/10.3145/epi.2018.mar.01

Goyanes, Manuel (2015). “¿Hacia una investigación estandarizada?”. Observatorio (OBS*), v. 9, n. 3, pp. 85-99. http://www.scielo.mec.pt/scielo.php?script=sci_arttext\&pid=S1646-59542015000300006

Goyanes, Manuel; Rodríguez-Gómez, Eduardo-Francisco (2018). “¿Por qué publicamos? Prevalencia, motivaciones y consecuencias de publicar o perecer". El profesional de la información, v. 27, n. 3, pp. 548-558.

https://doi.org/10.3145/epi.2018.may.08

Gross, Alan G.; Harmon, Joseph E.; Reidy, Michael (2002). Communicating science. The scientific article from the $17^{\text {th }}$ century to the present. New York: Oxford University Press. ISBN: 9780195134544

Harzing, Anne-Wil; Alakangas, Satu (2016). "Google Scholar, Scopus and the Web of Science: a longitudinal and cross-disciplinary comparison". Scientometrics, v. 106, n. 2, pp. 787-804.

https://doi.org/10.1007/s11192-015-1798-9

Heidari, Shirin; Babor, Thomas F.; De-Castro, Paola; Tort, Sera; Curno, Mirjam (2016). "Sex and gender equity in research: rationale for the Sager guidelines and recommended use". Research integrity and peer review, v. 1, n. 2.

https://doi.org/10.1186/s41073-016-0007-6

Infoadex (2020). Estudio Infoadex de la inversión publicitaria en España. Madrid: Infoadex. https://bit.ly/2Y3rJVI

Izquierdo-Iranzo, Patricia (2019): “Cómo investigan los estudiantes de los grados de Comunicación. El peso del historicismo epistemológico de Kuhn”. En: Mañas-Viniegra, Luis; De-Vicente-Domínguez, Aida-María. Contenidos audiovisuales, narrativas y alfabetización mediática. Madrid: McGrawHill, pp. 341-354. ISBN: 9788448618162

Izquierdo-Iranzo, Patricia; Gaitán-Moya, Juan-Antonio; Caffarel-Serra, Carmen (2020). “Mapa de la mujer como sujeto y objeto de la investigación en Comunicación en España”. Comunicació. Revista de recerca i d’anàlisi, v. 37, n. 1, pp. 31-52.

https://doi.org/10.2436/20.3008.01.189 
Jensen, Kathrine S. H. (2005). Women working in science, engineering and technology, higher education and industry: A literature review. Manchester: Informatics Research Institute, Salford University.

Jiménez-Contreras, Evaristo; De-Moya-Anegón, Félix; Delgado-López-Cózar, Emilio (2003). “The evolution of research activity in Spain. The impact of the National Commission for the Evaluation of Research Activity (CNEAI)". Research policy, n. 32, pp. 123-142.

https://doi.org/10.1016/S0048-7333(02)00008-2

Jones, Daniel E. (1998). “Investigación sobre Comunicación en España. Evolución y perspectivas”. ZER, v. 3, n. 5, pp. $13-51$. https://ojs.ehu.eus/index.php/Zer/article/view/17359

Jones, Daniel E.; Baró, Jaume (2000). Investigación sobre Comunicación en España. Aproximación bibliométrica a las tesis doctorales (1926-1998). Barcelona: Com Cat.

Marcos-Recio, Juan-Carlos; Martínez-Pestaña, María-Jesús; Blasco-López, María-Francisca (2012). “Producción y dirección de Tesis doctorales sobre publicidad en la universidad española (1971-010)". Revista española de documentación científica, v. 35, n. 3, pp. 433-452.

https://doi.org/10.3989/redc.2012.3.890

Orduña-Malea, Enrique; Martín-Martín, Alberto; Delgado-López-Cózar, Emilio (2016). "ResearchGate como fuente de evaluación científica: desvelando sus aplicaciones bibliométricas". El profesional de la información, v. 25, n. 2, pp. 303-310. https://doi.org/10.3145/epi.2016.mar.18

Pacios, Ana-Reyes; Vianello-Osti, Marina; Rodríguez-Bravo, Blanca (2016). "Transparency and access to information on research projects in Spanish public universities". El profesional de la información, v. 25, n. 5, pp. 721-729.

https://doi.org/10.3145/epi.2016.sep.02

Perceval, José-María; Fornieles-Alcaraz, Javier (2008). “Confucio contra Sócrates: la perversa relación entre la investigación y la acreditación". Anàlisi, n. 36, pp. 213-224.

https://www.raco.cat/index.php/Analisi/article/view/94533/0

Piñuel-Raigada, José-Luis; Lozano-Ascencio, Carlos; García-Jiménez, Antonio (eds.) (2011). Investigar la Comunicación en España. Madrid: Asociación Española de Investigación en Comunicación (AE-IC). ISBN: 9788469427132

Pusser, Brian; Marginson, Simon (2013). "University rankings in critical perspective". The journal of higer education, v. 84, n. 4, pp. 544-568.

https://doi.org/10.1080/00221546.2013.11777301

Repiso, Rafael; Orduña-Malea, Enrique; Aguaded, Ignacio (2019). “Revistas científicas editadas por universidades en Web of Science: características y contribución a la marca universidad”. El profesional de la información, v. 28, n. 4, e280405. https://doi.org/10.3145/epi.2019.jul.05

Rodríguez-Gómez, Eduardo-Francisco (2016). “Aportación científica de los congresos de la AEIC (2008-2014): reseña histórica y estado actual de la investigación en Comunicación”. Historia y comunicación social, v. 21, n. 1, pp. 115-137. https://doi.org/10.5209/rev_HICS.2016.v21.n1.52687

Rodríguez-Serrano, Aarón; Gil-Soldevilla, Samuel (coords.) (2018). Investigar en la era neoliberal. Visiones críticas sobre la investigación en Comunicación en España. Barcelona: Universitat Autònoma de Barcelona, Servei de Publicacions. ISBN: 9788417429355

Saperas-Lapiedra, Enric (2016). “Cuatro décadas de investigación comunicativa en España. Los procesos de institucionalización y de profesionalización de la investigación (1971-2015)". Anuario electrónico de estudios en comunicación social "Disertaciones", v. 9, n. 2, pp. 27-45.

https://doi.org/10.12804/disertaciones.09.02.2016.02

Túñez-López, Miguel (2013). “El índice h de la investigación en Comunicación en España, Portugal y Latinoamérica: Web of knowledge (WoS), Scopus y Google Scholar Metrics". Comunicación \& society, v. 26, n. 4, pp. 53-75.

https://revistas.unav.edu/index.php/communication-and-society/article/view/36060

Tur-Viñes, Victoria; Núñez-Gómez, Patricia (2018). “Grupos académicos españoles de investigación en Comunicación”. Comunicación \& society, v. 31, n. 4, pp. 173-192.

https://doi.org/10.15581/003.31.4.173-192

Unidad de Mujeres y Ciencia (2016). Nota informativa sobre evaluación de la Integración del Análisis de Género en la Investigación (IAGI). Madrid: Mineco.

https://bit.ly/36FBCej

Urabayen, Miguel (1994). "La investigación sobre comunicación social en España: panorama general”. En: Tercera trobada iberoamericana d'investigadors de la comunicació. Cultura i comunicació social: América Llatina i Europa Ibérica. Barcelona: Cedic, pp. 81-86. 